\title{
Geotecnologias aplicadas à espacialização de pontos de outorgas no Estado do Rio de Janeiro
}

\author{
Vladimir da Franca Fernandes ${ }^{1}$ \\ Juliana Vasconcellos Baptista ${ }^{2}$ \\ Suelen Oliveira Alpino Rodrigues ${ }^{3}$
}
${ }^{1}$ Geógrafo - Esp. em Geotecnologias - Esp. em Planejamento e Gestão Ambiental - Mestrando em Ciências - COPPE/UFRJ
INEA - Instituto Estadual do Ambiente, Campo de São Cristóvão n $137,3^{\circ}$ andar - São
Cristóvão, Rio de Janeiro, RJ
vladimir.inea@gmail.com
${ }^{2}$ Geógrafa - Esp. em Geotecnologias - Esp. em Planejamento e Uso do Solo Urbano -
Mestranda em Geografia - UFF
INEA - Instituto Estadual do Ambiente, Campo de São Cristóvão n ${ }^{\circ} 137,3^{\circ}$ andar - São
Cristóvão, Rio de Janeiro, RJ
juli.inea@gmail.com

\author{
${ }^{3}$ Licenciada em Geografia - Pós-Graduanda em Geotecnologias - UFF \\ INEA - Instituto Estadual do Ambiente, Campo de São Cristóvão n $137,3^{\circ}$ andar - São \\ Cristóvão, Rio de Janeiro, RJ \\ sualpino@gmail.com
}

\begin{abstract}
The use of Geo Technologies for analysis of spatial distribution of geographic phenomena has proved of great importance for the planning and land management. In the State of Rio de Janeiro, the State Institute for the Environment - INEA makes use of these tools in their various activities of licensing and management of natural resources. The grant of rights over water use is an important tool for water resources management enshrined in the Federal law 9.433, 1997 and its main objectives to ensure users access to water, to minimize conflicts between users and prevent negative environmental impacts to Bodies of Water. From this scenario the Department of Hydrology, Marginal Strips and Grant of INEA initiated the mapping of grants in the State of Rio de Janeiro through the systematization of information regarding the users of water resources by ensuring the modernization of procedures, efficiency and quality in decision.
\end{abstract}

Palavras-chave: geotecnologias, outorga do direito pelo uso da água, Estado do Rio de Janeiro

\section{Introdução}

A outorga do direito pelo uso da água é um dos instrumentos de gestão dos recursos hídricos previsto na Lei Federal $n^{\circ}$ 9433/1997 que institui a Política Nacional de Recursos Hídricos. No estado do Rio de Janeiro, através da Lei Estadual $n^{\circ}$ 3239/1999 e de suas regulamentações, este instrumento é mais detalhado, respeitando as especificidades do estado de modo que seus objetivos sejam alcançados.

O instrumento de outorga é necessário para o gerenciamento dos recursos hídricos, pois permite ao administrador (outorgante) organizar os usos e realizar o controle quali- 
quantitativo da água, e ao usuário (requerente) a necessária autorização para o uso da água, garantindo, assim, o efetivo exercício dos direitos de acesso à água.

A outorga funciona de maneira integrada com os demais Instrumentos de Gestão previstos na Política Nacional de Recursos Hídricos e é uma condição ao Licenciamento Ambiental.

Para a gestão de recursos hídricos, as geotecnologias se tornaram as principais ferramentas para análise de dados espaciais. Este conjunto de técnicas computacionais está relacionado com a coleta, armazenamento e tratamento de informações georreferenciadas, ou seja, informações que estão ligadas a uma posição específica no globo terrestre por meio de suas coordenadas. Seu principal objetivo é fornecer ferramentas computacionais para que os usuários analisem evoluções espaciais e temporais de fenômenos geográficos e suas interrelações. Destacam-se entre as geotecnologias a cartografia digital, o sensoriamento remoto, a aerofotogrametria e o Sistema de Posicionamento Global (GPS). Todas estas tecnologias estão intimamente ligadas e se inter-relacionam em um ambiente SIG (Sistemas de Informações Geográficas).

\section{Outorga pelo uso da água}

No Estado do Rio de Janeiro compete ao Instituto Estadual do Ambiente (INEA) a gestão dos recursos hídricos sob jurisdição estadual e, consequentemente, a outorga de direito de uso destes recursos. As políticas nacional e estadual de recursos hídricos são os principais marcos do arcabouço legal que estabelecem as diretrizes para gestão da água no estado.

Por que outorgar? Os principais objetivos da outorga são: garantir a todos os usuários o acesso à água visando o uso múltiplo e a preservação das espécies da fauna e flora endêmicas ou em perigo de extinção e minimizar os conflitos entre os diversos setores usuários, além de evitar impactos ambientais negativos aos corpos hídricos.

Estão sujeitos à outorga os seguintes usos dos recursos hídricos: derivação ou captação de parcela da água existente em um corpo de água, para consumo; extração de água de aqüífero; lançamento, em corpo de água, de esgotos e demais resíduos líquidos ou gasosos, tratados ou não, com o fim de sua diluição, transporte ou disposição final; aproveitamento dos potenciais hidrelétricos e outros usos que alterem o regime, a quantidade ou a qualidade da água existente em um corpo hídrico ${ }^{1}$.

Alguns usos independem de outorga como, por exemplo: o uso de recursos hídricos para a satisfação das necessidades de pequenos núcleos populacionais, ou o de caráter individual, para atender às necessidades básicas da vida, as derivações, captações, extrações, lançamentos e acumulações da água em volumes considerados insignificantes ${ }^{2}$.

Para que os objetivos da outorga sejam alcançados é de fundamental importância que as relações entre os usuários de água, a disponibilidade hídrica e outros fatores naturais possam ser mensurados e analisados, também, sob o ponto de vista de suas distribuições espaciais.

\section{Uso de geotecnologias na outorga}

Em uma análise espacial se avaliarmos os elementos de maneira isolada, considerando apenas sua natureza, sua estrutura ou as classes desses elementos, o resultado obtido não será diferente de uma simples descrição. De fato é a inter-relação existente nos elementos que

\footnotetext{
${ }^{1}$ De acordo com o Art. 22 da Lei Estadual no 3239/1999.

${ }^{2}$ Limites de uso considerados insignificantes: Derivações e captações com vazões até 0,4 litros por segundo e volume máximo diário de 34.560 litros, com seus efluentes correspondentes; Extrações de água subterrânea inferiores ao volume diário equivalente a 5.000 (cinco mil) litros e respectivos efluentes. (Lei Estadual 4.247/03 alterada pela Lei Estadual $5.234 / 2008)$.
} 
permite conhecê-los, isto é, "fatos isolados são abstrações e o que lhe dá concretude é a relação que mantêm entre si”. (SANTOS, 1982).

A utilização de um Sistema de Informações Geográficas (SIG's) nos permite trabalhar com um grande volume de dados, estabelecer relações hierárquicas entre diversas camadas ou níveis de informações, maior velocidade no acesso aos dados, diversas formas de saída de informação além de gerar subsídios para uma análise sistêmica da gestão de recursos hídricos.

Dentre as principais funcionalidades dos SIG's pode-se destacar a capacidade de associação de uma base de dados tabular à uma entidade gráfica, além de representá-la em um mapa. É importante ressaltar que ao representá-la em um mapa esta assume um par de coordenadas e, consequentemente, uma localização no espaço (georreferenciamento). Essas entidades gráficas podem ser representadas através de pontos, linhas ou polígonos.

Representar graficamente um elemento natural da paisagem (rio) através de uma entidade gráfica (linha) e associá-la a um banco de dados nos permite analisá-lo, assim como todo o sistema do qual ele faz parte, com uma riqueza de informações diretamente proporcional ao nível de informações contidas no banco de dados.

No ano de 2009, no Instituto Estadual do Ambiente - INEA/RJ, foi dado início ao mapeamento dos Pontos Outorgados em corpos d'água no ERJ a partir de um banco de dados georreferenciados desenvolvido no Serviço de Outorga pelo Uso da Água (SEAGUA). O SEAGUA é parte integrante da Gerência de Hidrologia, Faixas Marginais e Outorga que integra a Diretoria de Licenciamento Ambiental.

A menor unidade de identificação e localização da interferência que um usuário produz em um corpo hídrico é determinada através de um ponto. Este ponto de contato do usuário com o corpo hídrico, seja ele uma captação, lançamento, derivação ou poço é mapeado através de um par de coordenadas e é denominado como um ponto de interferência.

O georreferenciamento desses pontos está sendo feito a partir das informações prestadas pelo requerente no Cadastro Nacional de Recursos Hídricos (CNARH) que é parte integrante dos processos administrativos de solicitação de outorga. Esses processos são abertos pelos requerentes com objetivo de se regularizarem junto ao órgão gestor quanto ao uso da água. Durante a análise técnica algumas informações são consistidas e posteriormente armazenadas em um banco de dados próprio.

Para qualquer finalidade de uso das águas de um rio, lago ou mesmo de águas subterrâneas, deve ser solicitada a Outorga (autorização de uso) ao Poder Público. Assim, no Estado do Rio de Janeiro, qualquer interferência que se pretenda realizar na quantidade ou na qualidade das águas de um manancial necessita de uma autorização do INEA.

\section{Métodos e Resultados}

Para este artigo o recorte espacial utilizado é o Estado do Rio de Janeiro (ERJ) e o recorte temporal os meses de janeiro a setembro de 2009.

O banco de dados (Figura 1) foi modelado no Serviço de Outorga pelo Uso da Água e para tal foi utilizado o Microsoft ACCESS, software de armazenamento e recuperação de dados. A entrada dos dados no banco é feita através da digitação das informações constantes no CNARH e de outras geradas a partir da análise dos técnicos do SEAGUA. 


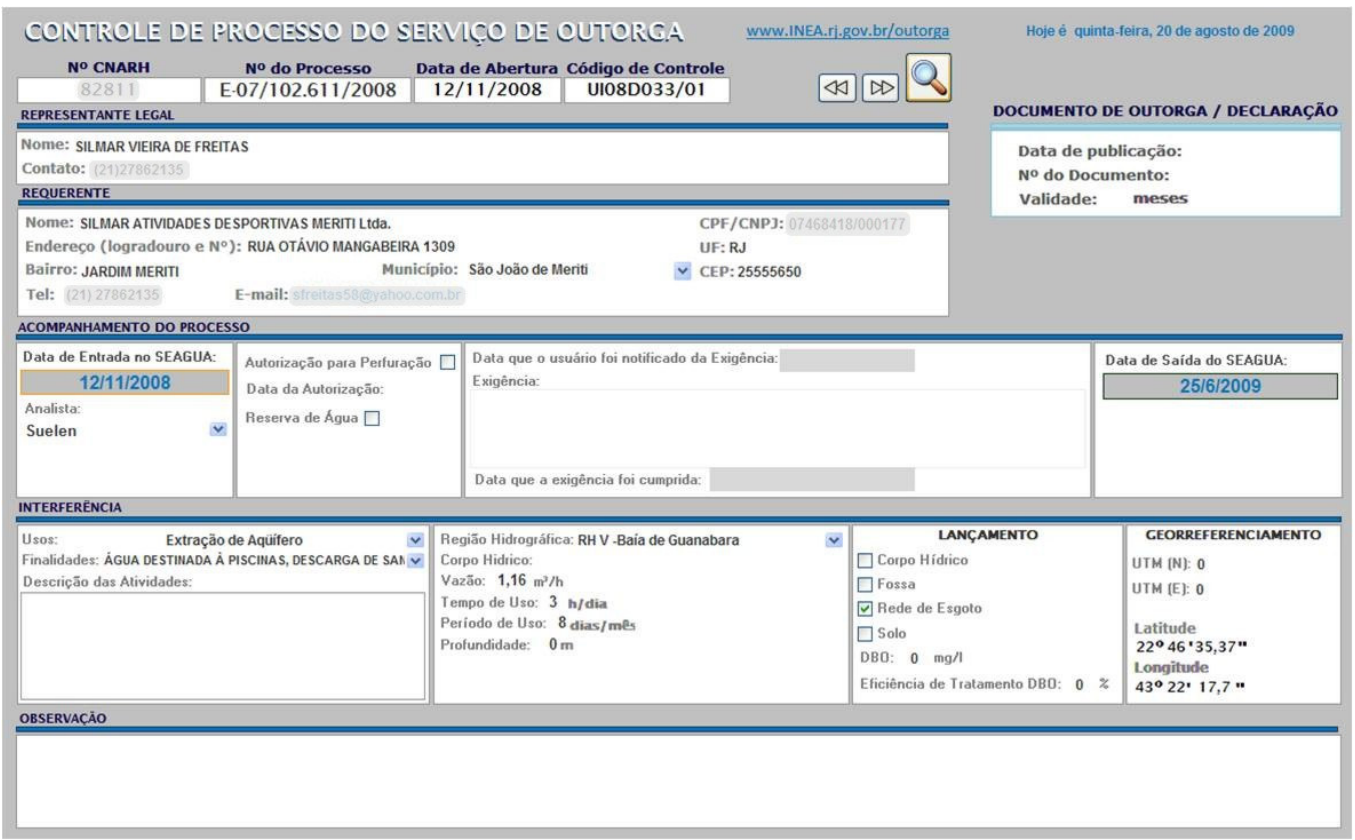

Figura 1: Exemplo do banco de dados - Cadastro de um ponto de interferência

Para o mapeamento e análises espaciais o software utilizado é o Arcgis 9.2. Este se mostrou apropriado, pois o Sistema Estadual de Recursos Hídricos do ERJ foi montado sobre a plataforma ARCGIS da empresa ESRI.

A partir dos dados obtidos foi possível elaborar o mapa de pontos outorgados no ERJ (Figura 2). Nele constam dois tipos de interferências: pontos outorgados e autorizações para perfuração de poços. Os pontos de outorgas estão relacionados ao uso do recurso hídrico e os pontos de autorização para perfuração estão relacionados à autorização para perfuração sem que haja uso da água. Caso a perfuração tenha sucesso e o perfurador encontre água disponível este dará entrada em processo de solicitação de outorga. Embora na autorização o recurso hídrico não esteja sendo usado para qualquer finalidade, esta foi considerada no mapeamento por se tratar de uma interferência em um corpo d'água (aqüífero) e que posteriormente será objeto de uma solicitação de outorga, conforme mencionado anteriormente.

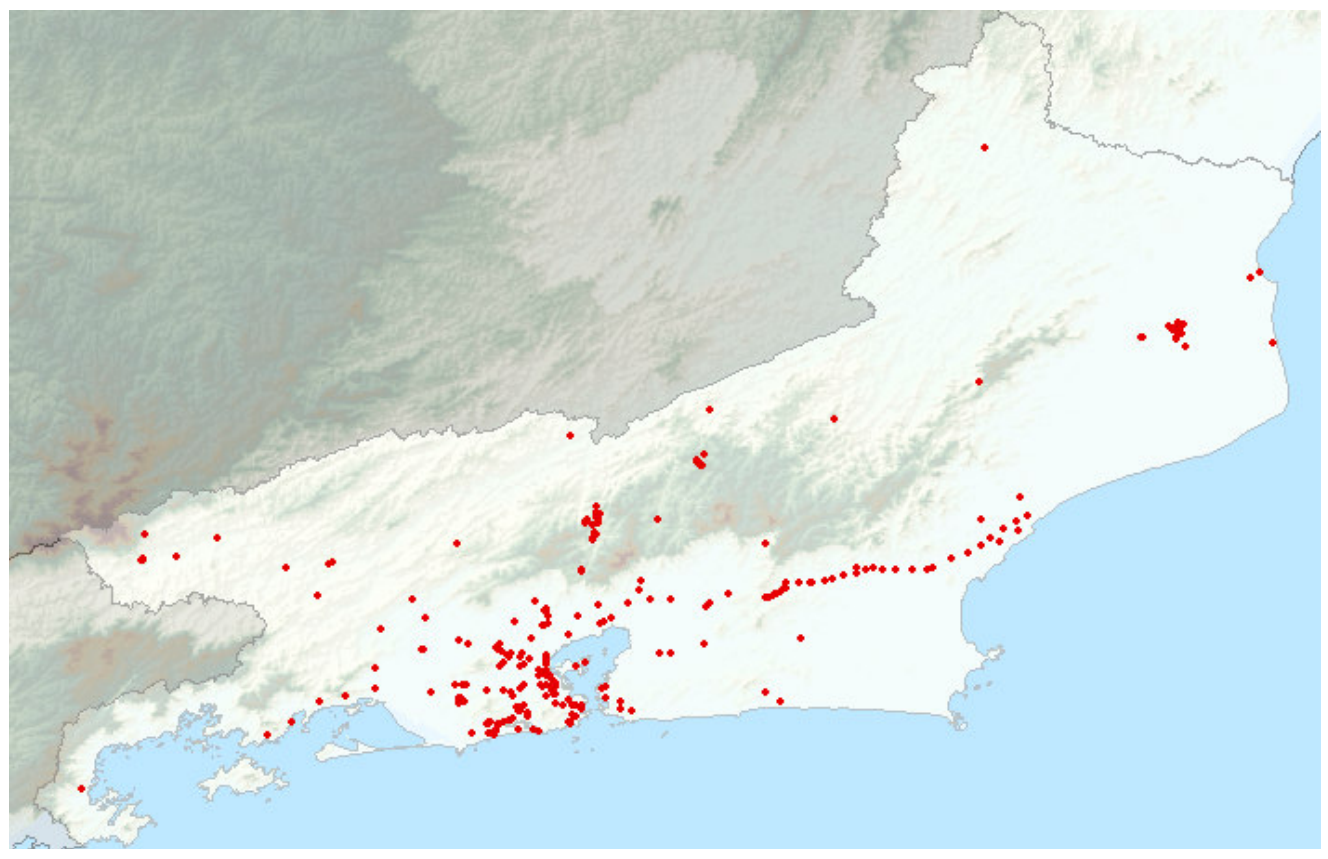

Figura 2: Pontos de interferência outorgados. Recorte temporal: Janeiro a setembro de 2009 
Outra forma de visualização da distribuição espacial desses dados é através do mapa de densidade a partir do método de Kernel (Figura 3). A concentração foi obtida somente a partir da proximidade entre os pontos de interferência $(20.000 \mathrm{~km}$ de raio).

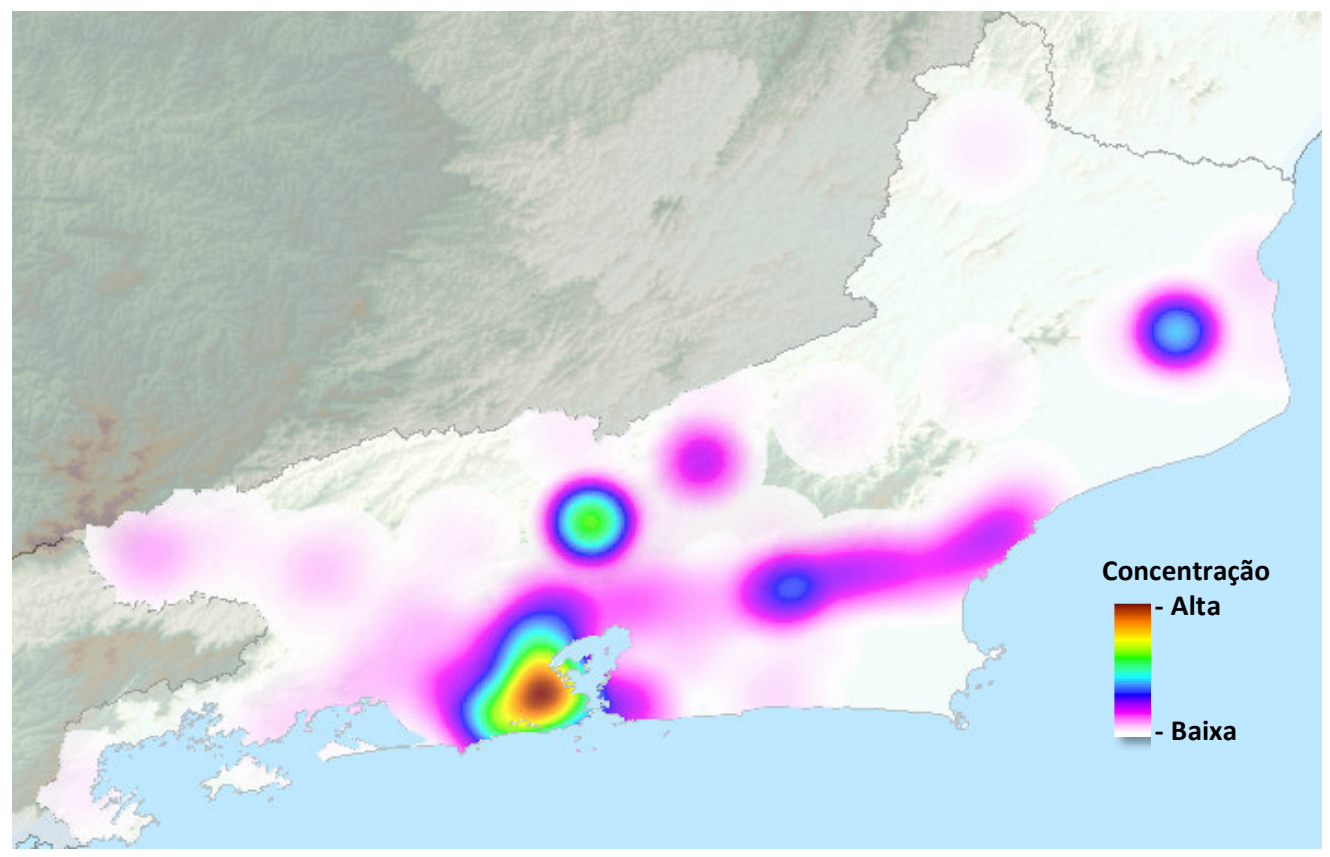

Figura 3: Mapa de densidade dos pontos de interferência outorgados.

A base de dados referente a cada ponto de interferência é composta por campos de informações, tais como: Vazão em m³/h, Profundidade do Poço, Coordenadas, Corpo Hídrico, Finalidade de Uso, Tipo de Interferência (Poço, Captação Superficial e Lançamento), Período Utilizado, Requerente, $\mathrm{N}^{\mathrm{o}}$ do Processo de Solicitação de Outorga, $\mathrm{N}^{\mathrm{o}} \mathrm{CNARH}$, Tipo de Documento, entre outros.

A partir desta base é possível gerar relatórios gerenciais e estatísticos, análises qualiquantitativas, espaciais e temporais.

Além dos mapas, é possível observar a distribuição dos dados de duas maneiras: a partir do mês de conclusão do processo administrativo, onde pode-se estabelecer uma relação entre a quantidade de pontos outorgados e a edição de um marco legal ou um evento hidrológico, por exemplo (Tabela 1) ou quantificar os pontos a partir da informação de Tipo de Documento (Tabela 2).

\begin{tabular}{c|c}
\hline MÊS & QUANTIDADE DE PONTOS \\
\hline Janeiro & 35 \\
\hline Fevereiro & - \\
\hline Março & 65 \\
\hline Abril & 14 \\
\hline Maio & 37 \\
\hline Junho & 24 \\
\hline Julho & 42 \\
\hline Agosto & 103 \\
\hline Setembro & 57 \\
\hline
\end{tabular}

Tabela 1: Quantidade de pontos outorgados por mês 


\begin{tabular}{l|c}
\multicolumn{1}{c|}{ Tipo } & QUANTIDADE DE PONTOS \\
\hline Alteração de Outorga & 3 \\
\hline Autorização para Perfuração & 51 \\
\hline Outorga & 161 \\
\hline Outorga Temporária & 12 \\
\hline Renovação de Outorga & 11 \\
\hline Reserva Hídrica & 6 \\
\hline Uso Insignificante & 133 \\
\hline Total & $\mathbf{3 7 7}$ \\
\hline
\end{tabular}

Tabela 2: Quantidade de interferências por tipo de solicitação

Para compor o mapeamento foram levantados dados cartográficos (bases cartográficas digitais, imageamento orbital de média e alta resolução espacial, levantamento aerofotogramétrico, etc). Esta base de dados que dá suporte às análises e produção de mapeamento básico e temático foi disponibilizada ao INEA e tem origem em diversos órgãos da administração pública e instituições de ensino e pesquisa (SERLA, IEF, FEEMA, CIDE, Prefeituras, Universidades, IBGE, EMBRAPA, etc).

\section{Conclusões}

Nos dias de hoje, a utilização de geotecnologias tornou-se indispensável para uma avaliação precisa dos diversos fenômenos geográficos que envolvam espaço e tempo, como por exemplo, o deslocamento de pessoas e mercadorias no território, o avanço desmatamento na Amazônia e o processo de urbanização nas grandes cidades.

A disponibilidade de softwares mais avançados junto com computadores com maior capacidade de armazenamento e velocidade de processamento tornou a utilização destas ferramentas cada vez mais acessíveis para um grande número de profissionais que atuam com planejamento e gestão.

As informações produzidas através de análises sobre a cartografia digital, imagens de satélite e fotografias aéreas trazem para o planejamento, em suas diversas escalas de análise, a possibilidade de melhor entender a dinâmica de produção e apropriação do espaço e os diversos fatores que nele interagem.

A partir do desenvolvimento deste artigo algumas considerações tornam-se relevantes, como por exemplo: os conceitos de Geotecnologias e Sistemas de Informações Geográficas, embora parecidos, não devem ser confundidos.

É importante ressaltar que as geotecnologias dão suporte aos SIG's e, por isso, precisam ter tratamento especial em suas precisões espacio-temporais.

O uso de Geotecnologias como suporte à gestão de recursos hídricos e ao licenciamento ambiental se mostrou de grande importância, pois possibilita a análise multidimensional e multi-escalar.

A distribuição espacial dos usuários de água no estado e a sistematização das informações a eles relacionadas formam a base que permeia os instrumentos de gestão de recursos hídricos no Estado do Rio de Janeiro. É importante tratar essas informações de maneira sistematizada, ou seja, de maneira que as diferentes formas de obtenção e armazenamento dos dados sejam organizadas para possibilitar uma melhor integração e compreensão destas informações. Estas devem sempre ficar ao alcance de um maior número de usuários para que as tomadas de decisão se tornem mais democráticas e participativas. 
O uso de Geotecnologias no mapeamento dos pontos outorgados no ERJ está contribuindo de maneira importante para modernização de procedimentos, agilidade e qualidade nas tomadas de decisão e, principalmente, na compreensão das interferências do homem na natureza por meio do uso da água.

\section{Referências bibliográficas}

CASTRO, C.M.; PEIXOTO, M. N.O. \& RIO, G. A. P. Riscos Ambientais e Geografia: Conceituações, Abordagens e Escalas. Environmental Risks and Geography: Conceptualizations, Approaches and Scales. Anuário do Instituto de Geociências UFRJ. Vol. 28-2 p. 11-30 - 2005.

FERNANDES, V. F.; VASCONCELLOS, J. Uso de Geotecnologias Aplicadas à Identificação e Análise de Áreas Susceptíveis a Risco de Enchentes e Deslizamentos: Bacia do Rio Jacaré, Niterói, RJ. 90 p. Monografia apresentado ao Departamento de Análise Geoambiental como requisito para obtenção do título de Especialista em Geotecnologias Aplicadas à Análise Ambiental de Bacias Hidrográficas - Instituto de Geociências, Universidade Federal Fluminense, Rio de Janeiro. 2004.

FERNÁNDEZ, M.A. Los gobiernos locales de América del Sur enfrentando la gestión de desastres. DIRDN - Informa, edição especial, 1996.

FOUCHER, M.. Esquisse d'une Géographie Humaine des Risques Naturels. Hérodote, 24: 40-67- 1982.

LEI FEDERAL Nº 9433 de 8 de janeiro de 1997. Institui a política nacional de recursos hídricos.

LEI ESTADUAL No 3239 de 2 de agosto de 1999. Institui a política estadual de recursos hídricos do Estado do Rio de Janeiro.

SANTOS, M. O Espaço e os seus elementos: questões de método. Revista Geografia e ensino. Belo Horizonte - MG, 1 (1): 19-30, março de 1982.

SILVA, P. P. L.; GUERRA, A. J. T.; DUTRA, L. E. D. Subsídios para avaliação econômica de impactos ambientais. In: CUNHA, S. B.; GUERRA, A. J. T. (Orgs.). Avaliação e perícia ambiental. Rio de Janeiro: Bertrand Brasil, 1999. 